\title{
MONOTONE CHROMATIC NUMBER OF GRAPHS
}

\section{ANWAR SALEH ${ }^{1, *}$, NAJAT MUTHANA ${ }^{1}$, WAFA AL-SHAMMAKH ${ }^{1}$, HANAA ALASHWALI ${ }^{2}$}

\author{
${ }^{1}$ Department of Mathematics, Faculty of Science, University of Jeddah, Jeddah, Saudi Arabia \\ ${ }^{2}$ Department of Mathematics, King Abdulaziz University, Jeddah, Saudi Arabia
}

${ }^{*}$ Corresponding author: asaleh1@uj.edu.sa

\begin{abstract}
For a graph $G=(V, E)$, a vertex coloring (or, simply, a coloring) of $G$ is a function $C: V(G) \rightarrow$ $\{1,2, \ldots, k\}$ (using the non-negative integers $\{1,2, \ldots, k\}$ as colors). In this research work, we introduce a new type of graph coloring called monotone coloring, along with this new coloring, we define the monotone chromatic number of a graph and establish some related new graphs. Basic properties and exact values of the monotone chromatic number of some graph families, like standard graphs, Kragujevac trees and firefly graph are obtained. Also, we get a characterization for bipartite graphs by defining the monotone bipartite graph. Exact values of the monotone chromatic number for some special case of Cartesian product of graphs are found. Finally, upper and lower bounds for monotone chromatic number of the Cartesian product for non trivial connected graphs are presented.
\end{abstract}

\section{INTRODUCTION}

Throughout this research work, by graph we mean finite graph without loops and parallel edges. Any notations or terminology not specifically defined here, we refer the book [6]. More details about coloring in graph and its related are reported in $([3,8])$. Two interesting types of coloring are introduced and studied in $([1,2,5])$. As usual, $P_{n}, C_{n}, K_{n}$ and $W_{n}$ are the $n$-vertex path, cycle, complete, and wheel graph, respectively, $K_{r, s}$ is the complete bipartite graph on $r+s$ vertices and $S_{r}$ is the star graph with $r+1$ vertices.

Received September $21^{\text {st }}, 2020$; accepted October $19^{\text {th }}, 2020$; published November $17^{\text {th }}, 2020$.

2010 Mathematics Subject Classification. 05C76, 05C22, 05C15.

Key words and phrases. monotone coloring; monotone chromatic number; monotone clique set; montone bipartite graph; complete monotone graph.

(C)2020 Authors retain the copyrights of their papers, and all open access articles are distributed under the terms of the Creative Commons Attribution License. 
Graph coloring is one of essential concepts in the theory of graphs. It has preoccupied a large number of people as a distraction puzzle during the 19th century and later in the framework of scientific research, since this conception exhibits a significant interest from a theoretical and practical point of view. Many applications are modeled and investigated with the use of graph coloring.

For a graph $\mathrm{G}=(\mathrm{V}, \mathrm{E})$, a vertex coloring (or, simply, a coloring) of $\mathrm{G}$ is a function $C: V(G) \rightarrow\{1,2, \ldots, k\}$ (using the non-negative integers $\{1,2, \ldots, k\}$ as colors).

The huge applications of coloring motivated us to introduce a new type of graph coloring called monotone coloring of the graph, we define the monotone chromatic number of a graph, complete monotone graph and monotone clique set of a graph. Some basic properties and relations with the other graph parameters and exact values of the monotone chromatic number of some graph families, like standard graphs, firefly graph and Kragujevac trees obtained. Also, we get a characterization for bipartite graphs by defining the monotone bipartite graph. Exact values of the monotone chromatic number for some special case of Cartesian product of graphs are found. Lastly upper and lower bounds for monotone chromatic number of the Cartesian product for non trivial connected graphs are presented.

\section{Monotone Chromatic Number of Graphs}

In this section, we define the monotone chromatic coloring and monotone chromatic number of a graph and give several preliminary results and straightforward facts regarding the monotone coloring of graphs. Also we found the monotone chromatic number for some families of graphs.

Definition 2.1. Let $G=(V, E)$ be a graph. A path $P=\left[v_{1}, v_{2}, \ldots, v_{k+1}\right]$ in $G$ is a monotone path if either $\operatorname{deg}\left(v_{i}\right) \leq \operatorname{deg}\left(v_{i+1}\right)$ or $\operatorname{deg}\left(v_{i}\right) \geq \operatorname{deg}\left(v_{i+1}\right)$ for all $i=1,2, \ldots, k$. Any two vertices $u$ and $v$ in $G$ are called monotone adjacent if there exists a monotone path connected them.

Definition 2.2. A monotone $k$ - coloring of the graph $G$ is coloring the vertices of $G$ with $k$ colors such that no two monotone adjacent vertices share the same color. The smallest integer $k$ such that $G$ has a monotone $k-k$ coloring is called the monotone chromatic number of $G$ and denoted by $\chi_{m o}(G)$. A graph $G$ is said to be monotone $k$ - colorable if it has monotone $k$ - coloring.

Monotone coloring as function we can define as:

Definition 2.3. The monotone coloring function is a function $f: V(G) \rightarrow 1,2,3, \ldots, k \subseteq \mathbb{N}$ which satisfy that for any two monotone adjacent vertices $v$ and $u, f(v) \neq f(u)$.

\section{Proposition 2.1.}


(1) For any path $P_{n}$ with $n \geq 2$ vertices,

$$
\chi_{m o}(G)= \begin{cases}2, & \text { if } n=2 ; \\ n-1, & \text { if } n \geq 3 .\end{cases}
$$

(2) For any connected regular graph $G$ with $n \geq 3$ vertices, $\chi_{m o}(G)=n$.

(3) For any complete bipartite graph $K_{r, s}$, where $1<r \leq s$, we have $\chi_{m o} K_{r, s}=2$.

(4) For any wheel graph $G \cong W_{r}$ with $r+1$ vertices, $\chi_{m o}(G)=r+1$.

(5) For any book graph $G \cong B_{m}$, we have $\chi_{m o}(G)=4$.

(6) For any helm graph $G \cong H_{n}$ with $2 n+1$ vertices, $\chi_{m o}(G)=n+2$.

(7) For any gear graph $G$ with $2 n+1$ vertices, we have

$$
\chi_{m o}(G)= \begin{cases}4, & \text { if } n=3 ; \\ 3, & \text { if } n \geq 4 .\end{cases}
$$

From the definition of the proper coloring and monotone coloring, it is obviously, that any monotone coloring is proper coloring but the converse is not true. As any two adjacent vertices in any graph are also monotone adjacent, then we have the following result.

Proposition 2.2. For any graph $G, \chi(G) \leq \chi_{m o}(G)$. The equality hold if and only if any two monotone adjacent vertices are adjacent.

\section{Proposition 2.3.}

(1) For any monotone $\chi_{m o}(G)$ - coloring of any graph $G$, all the very weak vertices has the same color.

(2) For any nontrivial connected graph $G$ with $n$ vertices, $2 \leq \chi_{m o}(G) \leq n$.

(3) For any graph $G \cong \cup_{i=1}^{r} G_{i}$, we have, $\chi_{m o}(G)=\max \left\{\chi_{m o}\left(G_{i}\right): i=1,2, \ldots r\right\}$.

Remark 2.1. Let $G$ and $H$ be any two graphs such that $H$ is subgraph of $G$. Then the monotone chromatic number of $G$ and $H$ are not comparable. That means all the possibilities allowed.

According to the monotone adjacency between vertices, we define the monotone bipartite graph, and complete monotone graph.

Definition 2.4. A bipartite graph $G$ is called monotone bipartite graph if and only if any monotone path in $G$ is of length at most one.

The definition of monotone bipartite graph characterize the trees into two families monotone trees and non-monotone trees The double star graph denoted by $B(r, s)$ with $r+s+2$ vertices, is a tree that containing exactly two non-pendent vertices. 
Proposition 2.4. For any double star graph $G \cong=B(r, s)$ with $r+s+2$ vertices, such that $r<s$, $\chi_{m o}(G)=3$.

Theorem 2.1. Let $G$ be any graph. Then $G$ is monotone bipartite graph if and only if $\chi_{m o}(G)=2$.

Proof. Let $G$ be a graph such that $\chi_{m o}(G)=2$ and suppose contrary that there is monotone path uvw of length 2 , we need at least three colors for monotone coloring of $G$ that means $\chi_{m o}(G) \geq 3$ which is a contradiction. Therefore any monotone is of length at most one. Hence $G$ is monotone bipartite graph. To prove the other direction, suppose, that $G$ is monotone bipartite graph. Then the result coming from the Proposition 2.2.

Corollary 2.1. For any monotone tree $T_{n}$ with $n$ vertices, $\chi_{m o}(G)=2$.

Proposition 2.5. For any connected graph $G$ with $n \geq 3$ vertices, $\chi_{m o}(G)=2$ If and only if for any vertex $v \in V(G)$, either $v$ is very strong or very weak.

Proof. Suppose that $\chi_{m o}(G)=2$, and assume in contrary that, there is a vertex $v \in V(G)$ which is neither very strong nor very weak. Then we have two cases:

Case 1 : if $\operatorname{deg}(v)=1$, then $v$ is very weak which contradict our assumption.

Case 2: if $\operatorname{deg}(v) \neq 1$, then $v$ must belongs to some path say uvw such that $\operatorname{deg}(u)<\operatorname{deg}(v)<\operatorname{deg}(w)$ or $\operatorname{deg}(u)=\operatorname{deg}(v)=\operatorname{deg}(w)$ or one of the vertices $u$, or $w$ has the same degree as $v$. For all of these cases, it needs at least 3 colors to monotone coloring which contradicts that $\chi_{m o}(G)=2$.

Now, suppose that for any vertex $v \in V(G)$, either $v$ is very strong and one very weak. So, any monotone path will contains only two vertices; one of them is very weak and the other is very strong. Hence $\chi_{m o}(G)=$ 2.

Corollary 2.2. A connected graph $G=(V ; E)$ is monotone bipartite graph if and only if any vertex $v \in V(G)$ is either very strong or very weak.

Proposition 2.6. For any connected graph $G$ with $n \geq 2$ vertices, $\chi_{m o}(G)=n$ If and only $G$ is complete monotone graph.

Proof. let $G$ be a monotone complete graph with $n$ vertices and suppose that $G$ in contrary not satisfy the condition $G$ contains at most either one very weak or one very weak vertex. Suppose the vertex set $V(G)=\left\{v_{1}, v_{2}, \ldots, v_{n}\right\}$, such that $v_{1}$ is very weak vertex, then if there is another very weak vertex say $v_{i}$ then there is no monotone vertex between $v_{1}$ and $v_{i}$ which is a contradiction with the definition of the monotone complete graph, similarly if there is very strong vertex say $v_{j}$, then there is no monotone path 
between $v_{1}$ and at least one of the neighborhood of the vertex $v_{j}$. The proof in the same way if we suppose $v_{1}$ is very strong. Hence $G$ contains at most either one very weak or one very weak vertex.

To prove the other direction. Suppose $G$ contains at most either one very weak or one very strong vertex. then clearly there exist a monotone path containing all the vertices that means between any two vertices there is a monotone. Hence $G$ is monotone complete graph.

Definition 2.5. A subset $X \subseteq V(G)$ of size $k$ is called a monotone $k$ - clique of $G$ if between any two vertices $u$ and $v$ in the set $X$ there is a monotone path. The monotone clique number of $G$ denote by $\omega_{m o}(G)$ is the largest positive integer $k$ such that $G$ contains a monotone $k-$ clique. The monotone clique set with size $\omega_{m o}(G)$ is called maximum monotone clique.

Definition 2.6. ([4]) Let $P_{3}$ be the 3 - vertex tree, rooted at one of its terminal vertices. For $k=2,3, \cdot, k$; construct the rooted tree $B_{k}$ by identifying the roots of $k$ copies of $P_{3}$. The vertex obtained by identifying the roots of $P_{3}$-trees is the root of $B_{k}$.

Definition 2.7. ([4]) Let $d \geq 2$ be an integer.Let $\beta_{1}, \beta_{2} 1, \cdots, \beta_{d}$, be rooted trees, specified in Definition 2.6, i.e., $\beta_{1}, \beta_{2}, \cdots, \beta_{d} \in\left\{B_{2} . B_{3}, \ldots\right\}$. A Kragujevac tree $T$ is a tree possessing a vertex of degree $d$, adjacent to the roots of $\beta_{1}, \beta_{1}, \cdots, \beta_{d}$. This vertex is said to be the central vertex of $T$, whereas $d$ is the degree of $T$.

Theorem 2.2. Let $G \cong \boldsymbol{K g}_{d, k}$, where $\boldsymbol{K}_{d, k}$ is the Kragujevac tree of degree $d \geq 2$ and with branches $B_{K_{i}}$ $, i=1,2, \ldots$, where every branch $B_{K_{i}}$ contains $k_{i}$ pendant vertices and if $t$ is the number of branches $B_{K_{i}}$ where $d=k_{i}$. Then

$$
\chi_{m o}(G)= \begin{cases}3, & \text { if the central vertex is very weak vertex; } \\ 4, & \text { if the central vertex is very strong vertex; } \\ 5, & \text { if the central vertex is very typical vertex; } \\ d+3, & \text { if the central vertex is regular vertex; } \\ t+4, & \text { if the central vertex is weak or strong vertex } \\ t+5, & \text { if the central vertex is typical vertex. }\end{cases}
$$

Proof. Let $G$ be the Kragujevac tree $\mathbf{K g}_{d, k}$ of degree $d \geq 2$ with enteral vertex $v$ and with branches $B_{K_{i}}$ $, i=1,2, \cdots$, where every branch $B_{K_{i}}$ contains $k_{i}$ pendant vertices and if $t$ is the number of branches $B_{K_{j}}$ where $d=k_{j}$. we have 7 possibilities for the type of the central vertex $v$ of the Kragujevac tree:

Case 1. The central vertex $v$ is very strong, in this case, we can define monotone coloring function by partition the vertex set of the tree into 4 classes, first class, the pendant vertices, second class the support vertices and third class the roots vertices of the branches and fourth class the central vertex and give color for each class, that means $\chi_{m o}(G) \leq 4$ and it is not difficult to see that, any set contains the the pendent 
vertex with its support vertex and the root vertex in any branch along with the central vertex $v$ is clique set with 4 vertices. That means in this case $\chi_{m o}(G)=4$.

Case 2. The central vertex $v$ is very weak, then we can define monotone coloring function $C_{m o}$ by partition the vertex set into 4 subsets, $S_{1}$ is the pendant vertices, $S_{2}$ the support vertices and $S_{3}$ be the roots vertices of the branches and $S_{4}$ be the central vertex $v$ and giving color for each subset as following:

$$
C_{m o}(x)= \begin{cases}i, & \text { if } x \in S_{i} \text { and } x \text { not the central vertex; } \\ 1 \text { or } 2, & \text { if } x \text { is the central vertex. }\end{cases}
$$

Therefore, $\chi_{m o}(G) \leq 3$ and since the set of vertices in any path between the pendant vertex of any branch and its root vertex is monotone clique set. Hence in this case $\chi_{m o}(G)=3$.

Case 3. The central vertex $v$ is regular vertex, then in this case, let us partition the vertex set into three subsets; the set of pendant vertices $S_{1}$, the support vertices $S_{2}$ and the set of roots vertices with the central vertex $S_{4}$, we define a monotone coloring function which assign $d+1$ different colors to the vertices of $S_{3}$ and one color to all the vertices of $S_{2}$ and another different color to the vertices of $S_{1}$, so, we need to $d+3$ colors to this monotone coloring function, therefore $\chi_{m o}(G) \leq d+3$. Obviously, if we take any pendant vertex along with its support vertex in any branches along with the set $S_{3}$ will make monotone clique set of $G$ of size $d+3$. Hence $\chi_{m o}(G)=d+3$.

Case 4. The central vertex $v$ is weak vertex, in this case, let us partition the vertex set into the following subsets of vertices, $S_{1}$, the set of pendant vertices, $S_{2}$ the set of support vertices, $S_{3}$ the central vertex and the set of root vertices of degree equal to $d$ and $S_{4}$ the set of root vertices with degree greater than $d$.

Now, $\left|S_{3}\right|=t+1$, we define monotone coloring function by assigning one color say 1 to the vertices of $S_{1}$ and another color say 2 to the vertices of $S_{2}$ and assign $t+1$ different colors to the $t+1$ vertices in $S_{3}$ and assign one other color to the vertices in $S_{4}$. So we need to $t+4$ colors in this monotone coloring function and therefore, $\chi_{m o}(G) \leq t+4$.

Also, it is obviously to see that the vertices of $S_{3}$ with one pendent vertex with its support vertex and one root vertex from $S_{4}$ will make clique set in $G$. Hence $\chi_{m o}(G)=t+4$.

Case 5. The central vertex $v$ is strong vertex, in this case let us partition the vertex set into the following subsets of vertices; $S_{1}$ the set of pendant vertices, $S_{2}$ the set of support vertices $S_{3}$ the central vertex with the set of root vertices of degree equal to $d$ and $S_{4}$ the set of root vertices with degree less than $d$. Now, if the number of root vertices with degree $d$ is $t$, we define monotone coloring function by assigning one color say 1 to the vertices of $S_{1}$ and another color say 2 to the vertices of $S_{2}$ and assign $t+1$ different colors to the $t+1$ vertices in $S_{3}$ and assign one other color to the vertices in $S_{4}$. So we need to $t+4$ colors in this monotone coloring function and therefore, $\chi_{m o}(G) \leq t+4$. Also, obviously the set of vertices in $S_{3}$ with the pendant vertex and its support vertex from any branch $B_{k_{i}}$ with $k_{i}=d$ and the root vertex of any other branch $B_{k_{i}}$ with $k_{i} \leq d$ make a clique set in $G$ with size $t+4$. Hence $\chi_{m o}(G)=t+4$. 
Case 6. The central vertex $v$ is very typical vertex. Suppose there are $s$ root vertex with degree less than $d$. Let $S_{1}$ be the set of pendant vertices, $S_{2}$ the set of support vertices $S_{3}$ the set of roots vertices with degree less than $d$ and let $S_{4}$ be the root vertices of degree greater than $d$ and $S_{5}$ contains the central vertex. We can define monotone coloring function by assigning for any vertex $x \in S_{i}$ the color $i ; C_{m o}(x)=i$. That means $\chi_{m o}(G) \leq 5$. Also the set which contains one vertex along with its support vertex and the root vertex in the same branch $B_{k_{i}}$ where $k_{i} \leq d$ and the central vertex and one root vertex from any branch $B_{k_{i}}$ where $k_{i} \geq d$ is clique set of size 5 . Hence in this case $\chi_{m o}(G)=5$.

Case 7. The central vertex $v$ is typical vertex. Suppose there are $t$ root vertex with degree equal to $d$. Let $S_{1}$ be the set of pendant vertices, $S_{2}$ the set of support vertices $S_{3}$ the set of roots vertices with degree equal to $d$ and let $S_{4}$ be the root vertices of degree greater than $d$ and $S_{5}$ be the roots vertices of degree less than $d, S_{6}$ contains the central vertex. We construct monotone coloring function by assigning one color to the vertices in $S_{1}$ and another different color to the vertices in $S_{2}$ and $t+1$ different colors for the vertices in $S_{3}$ and $S_{6}$ also one other different color to the vertices in $S_{4}$ and one different color to the vertices in $S_{5}$. Therefore $\chi_{m o}(G) \leq t+5$.

Now, we have clique set of size $t+5$ which contains one pendant vertex, support vertex and root vertex from any of the branch $B_{k_{i}}$, where $k_{i}<d$ and the $t$ vertices of $S_{3}$ along with the central vertex, one root vertex from any branch $B_{k_{i}}$, where $k_{i}>d$. Hence $\chi_{m o}(G)=t+5$.

Theorem 2.3. For any nontrivial connected graph $G$, with monotone chromatic number $\chi_{\text {mo }}(G)$, there exist at least one maximum monotone clique set with size $\omega_{m o}(G)=\chi_{m o}(G)$.

Proof. Let $G$ be any nontrivial connected graph with monotone chromatic number $\chi_{m o}(G)=s$. Suppose to the contrary, that we have maximum clique set $A$ with size $|A|=\omega_{m o}(G)=t$, where either $t<s$ or $t>s$.

Case 1. If $t<s$. Then there exists at least one coloring class say $B$ and at least one monotone path between every two elements one from $A$ and one from $B$ which is contradict that $A$ is the monotone clique with maximum size.

Case 2. If $t>s$, then at least we need to $t+1$ colors for monotone coloring which is contradict that $\chi_{m o}(G)=s$. Hence, $\omega_{m o}(G)=\chi_{m o}(G)$.

Theorem 2.4. Let $G \cong K_{m_{1}, m_{2} \cdots m_{k}}$, where $m_{1} \leq m_{2} \leq \cdots \leq m_{k}$ be any complete $k$ - partite graph and there are $t_{i}$ partite sets of the same number of vertices $\lambda_{i}$, where $i=1,2, \cdots, s$ for some positive integer $i$. Then

$$
\chi_{m o}(G)=k+\sum_{i=1}^{s} t_{i}\left(\lambda_{i}-1\right) .
$$

Proof. Let $G \cong K_{m_{1}, m_{2} \cdots m_{k}}$, where $m_{1} \leq m_{2} \leq \cdots \leq m_{k}$ be any complete $k$ - partite graph and there are $t_{i}$ partite sets of the same number of vertices $\lambda_{i}$, where $i=1,2, \cdots, s$ for some positive integer $i$, by reordering 
the partite sets which they have different number of elements as

$$
V_{1}, V_{2}, \cdots V_{k-\sum_{i=1}^{s} t_{i}}
$$

we can define a monotone coloring function as the following.

$$
f: V(G) \rightarrow\left[k+\sum_{i=1}^{s} t_{i}\left(\lambda_{i}-1\right)\right],
$$

by assigning the color $i$ for each vertex in $V_{i}$ and assigning for each vertex in the equal parite sets to different colors that means assign $\sum_{i=1}^{s} t_{i} \lambda_{i}$ different colors to the vertices in

$$
V-\bigcup_{i=1}^{\sum_{i=1}^{s} t_{i}} V_{i}
$$

Clearly, the function $f$ which defined above is monotone coloring function on $G$. Therefore,

$$
\chi_{m o}(G) \leq k+\sum_{i=1}^{s} t_{i}\left(\lambda_{i}-1\right) .
$$

It is not difficult to check that the set which contains the vertices in

$$
\bigcup_{i=1}^{\sum_{i=1}^{s} t_{i}} V_{i}
$$

and only one vertex from each $V_{i}$ will make clique set with $k+\sum_{i=1}^{s} t_{i}\left(\lambda_{i}-1\right)$. Thus,

$$
\chi_{m o}(G) \geq k+\sum_{i=1}^{s} t_{i}\left(\lambda_{i}-1\right) .
$$

Hence by inequalities 2.1 and 2.2 , we get,

$$
\chi_{m o}(G)=k+\sum_{i=1}^{s} t_{i}\left(\lambda_{i}-1\right) .
$$

Theorem 2.5. Let $G$ be any connected graph with $n \geq 3$ vertices and $D$ is the set of distinct degrees of the vertices. Then $G$ is monotone complete graph if and only if for any set of vertices with the same degree in $G$ is clique set in $G$.

Proof. Let $G$ be monotone complete graph with $n \geq 3$ vertices, since $G$ is monotone complete, then $\omega_{m o}(G)=$ $n$. Therefore $V(G)$ is a monotone clique set contains all the vertices of $G$.

If $G$ is regular, then there is only one clique set containing all the vertices. Suppose the graph is not regular that means the set $D$ is of size greater than or equal to 2 . Let $D=\left\{k_{1}, k_{2}, \ldots, k_{t}\right\}$ for some integer $t \geq 2$, also let $C_{1}, C_{2}, \ldots, C_{t}$ be the sets of vertices of degrees $k_{1}, k_{2}, \ldots, k_{t}$ respectively. Suppose in contrary, that there exists one set $C_{j}, 1 \leq j \leq t$ such that $C_{j}$ not a monotone clique set, then there exist at least two vertices $u$ and $v$ which they are not monotone adjacent in $G$ which is a contradict that $V(G)$ is clique set. Similarly, let $C_{1}, C_{2}, \ldots, C_{t}$ are monotone clique sets in $G$. suppose $G$ is not monotone complete, then there 
exist at least two vertices $u$ and $v$ not monotone adjacent if they are with the same degree, then they will belong to same set $C_{j}, 1 \leq j \leq t$ which is a contradiction, similarly we will get a contradiction even if they have different degrees.

We recall, that in [7], a firefly graph $F_{s, t, l}$ where $s \geq 0, t \geq 0, n-2 s-2 t-1 \geq 0$ is a graph of order $n$ that consists of $s$ triangles, $t$ pendent paths of length 2 and 1 pendant edges sharing a common vertex (see Figure 1).

Proposition 2.7. For any firefly graph $G \cong F_{s, t, l}, \chi_{m o}(G)=3$.

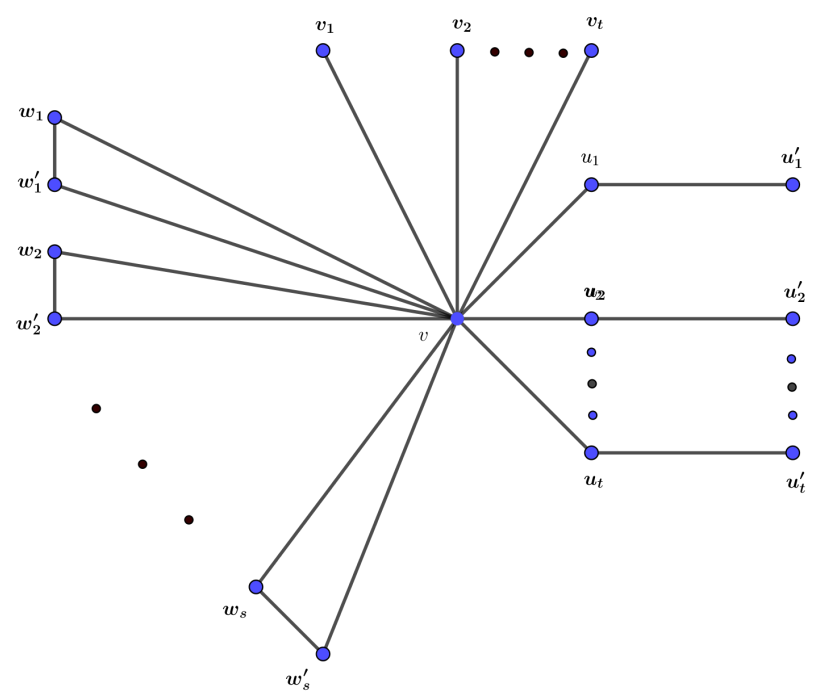

FIGURE 1. Firefly graph

Proof. Let $G \cong F_{s, t, l}$ where $s \geq 0, t \geq 0, n-2 s-2 t-1 \geq 0$ be a firefly graph and by labeling the vertices as in Figure 1. By partition the vertex set into the following sun- sets of vertices, $S_{1}=\left\{w_{1}, w_{2}, \ldots, w_{s}\right\}$, $S_{2}=\left\{w_{1}^{\prime}, w_{2}^{\prime}, \ldots, w_{s}^{\prime}\right\}, S_{3}=\left\{v_{1}, v_{2}, \ldots, v_{l}\right\}, S_{4}=\left\{u_{1}, u_{2}, \ldots, u_{t}\right\}, S_{5}=\left\{u_{1}^{\prime}, u_{2}^{\prime}, \ldots, u_{t}^{\prime}\right\}$ and $S_{6}$ is the central vertex. By defining the monotone coloring function $f: V(G) \longrightarrow\{1,2,3\}$ as following,

$$
f(x)= \begin{cases}1, & \text { if } x \in S_{1} \cup S_{3} \cup S_{4} ; \\ 2, & \text { if } x \in S_{2} \cup S_{5} ; \\ 3, & x \text { is the central vertex. }\end{cases}
$$

Therefore, $\chi_{m o}(G) \leq 3$. The set of vertices in any triangle in the firefly graph generate a monotone clique set, that means $\chi_{m o}(G) \geq 3$. Hence, $\chi_{m o}(G)=3$. 
The corona product $G_{1} \circ G_{2}$ of two graphs $G_{1}$ and $G_{2}$, where $V\left(G_{1}\right), V\left(G_{2}\right)$ are the set of vertices of $G_{1}, G_{2}$ respectively, is the graph obtained by taking $\left|V\left(G_{1}\right)\right|$ copies of $G_{2}$ and joining each vertex of the $i$-th copy with the corresponding vertex $u \in V\left(G_{1}\right)[6]$.

Proposition 2.8. For any positive integer $a \geq 3$, there exists a graph $G$ such that $\chi(G)=a$ and $\chi_{m o}(G)=$ $a+1$.

Proof. Let $G$ be a graph which construct by the corona product between the complete graphs $K_{a}$ and $K_{1}$. That means $G \cong K_{a} \circ K_{1}$ where $a \geq 3$. Then it is not difficult to see that $\chi(G)=a$ and $\chi_{m o}(G)=a+1$.

Definition 2.8. A subset $S \subseteq V$ is called monotone independent set, if the set $S$ does not contains any monotone adjacent vertices. The maximum cardinality of the monotone independent set is called monotone independence number of the graph and denoted by $\beta_{m o}(G)$. For example; for any path $P_{n}$ with $n \geq 3$ vertices, $\beta_{m o}\left(P_{n}\right)=2$. The monotone independence number of any complete monotone graph is one.

Theorem 2.6. Let $G$ be a graph with $n$ vertices and with monotone independence number $\beta_{m o}(G)$. Then

$$
\chi_{m o}(G) \geq \frac{n}{\beta_{m o}(G)}
$$

Further, the equality holds if $G$ is complete monotone graph.

Proof. Let $\chi_{m o}(G)=k$ and let $C_{m o}: V(G) \longrightarrow 1,2, \ldots, k$ be monotone k-coloring function. Let $\beta_{m o}(G)$ be the monotone independence number of G. We define $V_{j}=\left\{v \mid C_{m o}(v)=j\right\}$ for $j=1,2, \ldots, k$. Obviously, $V_{j}$ is monotone independent set and clearly $\left|V_{j}\right| \leq \beta_{m o}(G)$ and

$$
\sum_{j=1}^{k}\left|V_{j}\right| \leq k \beta_{m o}(G)=\chi_{m o}(G) \beta_{m o}(G) .
$$

Also since

$$
\sum_{j=1}^{k}\left|V_{j}\right|=n
$$

Thus, from inequalities 2.3 and 3.1

$$
n \leq \chi_{m o}(G) \beta_{m o}(G) \Rightarrow \chi_{m o}(G) \geq \frac{n}{\beta_{m o}(G)} .
$$

Also, if $G$ is complete monotone graph, then $\beta_{m o}(G)=1$.

Therefore,

$$
\chi_{m o}(G)=\frac{n}{\beta_{m o}(G)}=n
$$




\section{Monotone Chromatic number for Some Cartesian Product of Graphs}

The Cartesian product $G_{1} \square G_{2}$ of two graphs $G_{1}$ and $G_{2}$, where $V\left(G_{1}\right), E\left(G_{1}\right)$ and $V\left(G_{2}\right), E\left(G_{2}\right)$ are the sets of vertices and edges of $G_{1}$ and $G_{2}$, respectively, has the vertex set $V\left(G_{1}\right) \times V\left(G_{2}\right)$ and two vertices $\left(u, u^{\prime}\right)$ and $\left(v, v^{\prime}\right)$ are connected by an edge if and only if either $\left(u=v\right.$ and $\left.u^{\prime} v^{\prime} \in E\left(G_{2}\right)\right)$ or $\left(u^{\prime}=v^{\prime}\right.$ and $\left.u v \in E\left(G_{1}\right)\right)[6]$.

Theorem 3.1. Let $G \cong P_{r} \square P_{s}$ where $r, s \geq 3$. Then

$$
\chi_{m o}(G)=(r-2)(s-1)+1 .
$$

Proof. Let $G \cong P_{r} \square P_{s}$ where $r, s \geq 3$. By labeling the vertices of the graph $G$ from the left to the right the first line $L_{1}$ of the vertices by $v_{1,1}, v_{1,2}, \ldots, v_{1, s-1}, v_{1, s}$, the second line of the vertices $v_{2,1}, v_{2,2}, \ldots, v_{2, s-1}, v_{2, s}$ and so on till line $L_{r-1}$ by $v_{r-1,1}, v_{r-1,2}, \ldots, v_{r-1, s-1}, v_{r-1, s}$ and finally, the vertices of the line $L_{r}$ by $v_{r, 1}, v_{r, 2}, \ldots, v_{r, s-1}, v_{r, s}$. let us make partition for the vertex set to the following subsets

$$
\begin{aligned}
S_{1} & =\left\{v_{1,1}, v_{1, s}, v_{r, 1}, v_{r, s}\right\} . \\
S_{2} & =\left\{v_{i, j}: 1<i<r, 1 \leq j<s\right\} . \\
S_{3} & =\left\{v_{1, j}, v_{r, j}: 1<j<s\right\} . \\
S_{4} & =\left\{v_{i, s}: 1<i<r\right\} .
\end{aligned}
$$

We can define a monotone coloring function as following

$$
f\left(v_{i, j}\right)= \begin{cases}1, & \text { if } v_{i, j} \in S_{1} \\ (i-2) s+j+1, & \text { if } v_{i, j} \in S_{2} \\ f\left(v_{j}, 1\right), & \text { if } v_{i, j} \in S_{3} \\ f\left(v_{j}, 1\right), & \text { if } v_{i, j} \in S_{4} .\end{cases}
$$

Clearly $f$ is a monotone-k-coloring function, where $k=(r-2)(s-1)+1$. So

$$
\chi_{m o}(G) \leq(r-2)(s-1)+1 .
$$

The set $S_{2} \cup\left\{v_{1,1}\right\}$ is monotone clique set with $(r-2)(s-1)+1$ vertices. Therefore,

$$
\chi_{m o}(G) \geq(r-2)(s-1)+1 .
$$

Hence by inequalities 3.1 and 3.2 , we get,

$$
\chi_{m o}(G)=(r-2)(s-1)+1 .
$$


Theorem 3.2. Let $G \cong C_{r} \square P_{s}$, where $r, s \geq 3$. Then

$$
\chi_{m o}(G)=r(s-1) .
$$

Proof. Let $G \cong C_{r} \square P_{s}$, where $r, s \geq 3$. By labeling the vertices of the internal and external cycles of $G$ as the vertices of the internal cycle by $v_{1}, v_{2}, \ldots, v_{r}$ and the vertices of external cycle by $u_{1}, u_{2}, \ldots, u_{r}$ see as Figure 2. Let us partition the set of vertices as following subsets:

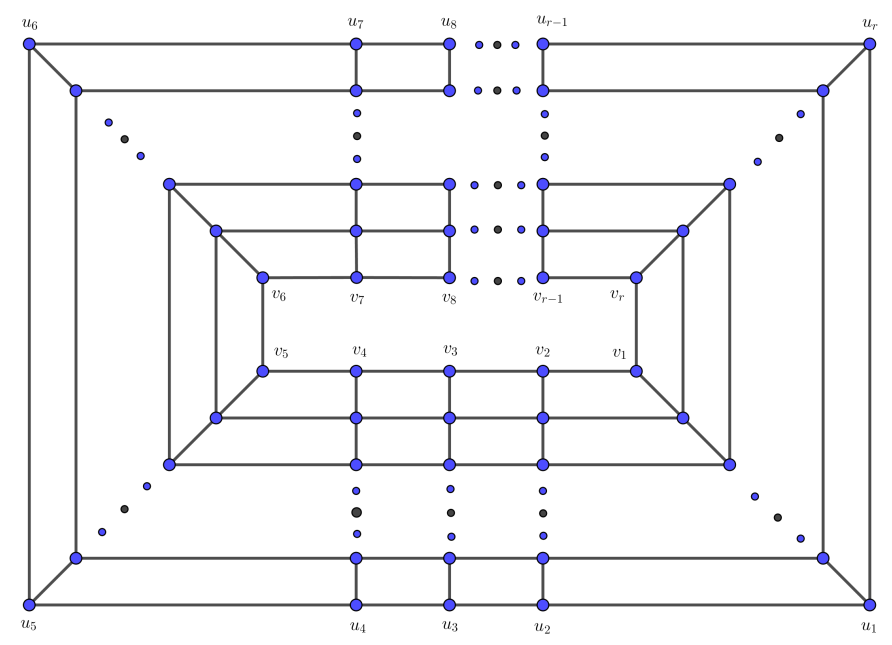

Figure 2. $C_{r} \square P_{s}$

$$
\begin{aligned}
& S_{1}=\left\{v_{i}, 1 \leq i \leq r\right\} . \\
& S_{2}=\left\{u_{i}, 1 \leq i \leq r\right\} . \\
& S_{3}=\left\{w_{j}, w_{j} \in V(G) \text { and } w \notin\left(S_{1} \cup S_{2}\right)\right\} .
\end{aligned}
$$

By defining a the coloring function, $f: V(G) \longrightarrow\{1,2, \ldots, r(s-1)\}$ such that: for any vertex $x$

$$
f(x)= \begin{cases}i, & \text { if } x=v_{i} \text { or } x=u_{i} ; \\ j+r, & \text { if } w_{j} \in S_{3} .\end{cases}
$$

Obviously, $f$ is a monotone $r(s-1)$ coloring function. So

$$
\chi_{m o}(G) \leq r(s-1)
$$

The set $S_{1} \cup S_{3}$ is monotone clique set with $r(s-1)$ vertices. Therefore,

$$
\chi_{m o}(G) \geq r(s-1) .
$$


Hence by inequalities 3.3 and 3.4, we get,

$$
\chi_{m o}(G)=r(s-1) .
$$

The stacked book is defined as the graph which construct by Cartesian product between star $S_{r}$ of $r+1$ vertices and path $P_{s}$ of $s$ vertices and denoted by $B_{r, s}$. That means $B_{r, s} \cong S_{r} \square P_{s}$.

Theorem 3.3. For any stacked book graph $G \cong S_{r} \square P_{s}$, where $r \geq 3$ and $s \geq 2$

$$
\chi_{m o}(G)= \begin{cases}4, & \text { if } s=2 \\ 2 s-3, & \text { if } s \geq 3\end{cases}
$$

Proof. Let $G \cong S_{r} \square P_{s}$. By labeling the vertices of the graph by using the horizontal lines of the vertices, let the center line $L_{0}$ and the outside lines are $L_{1}, L_{2}, \ldots, L_{r}$. By labeling the vertices of lines from the left to the right according to the line, the vertices on the center line means the vertices on the line $L_{0}$ are labeling as $v_{01}, v_{02}, \ldots, v_{0 s}$, the vertices on the line $L_{1}$ are labeling as $v_{11}, v_{12}, \ldots, v_{1 s}$, the vertices on the line $L_{2}$ are labeling as $v_{21}, v_{22}, \ldots, v_{2 s}$ and so on till the vertices on the line $L_{r}$ are labeling as $v_{r 1}, v_{r 2}, \ldots, v_{r s}$ (See Figure 3).

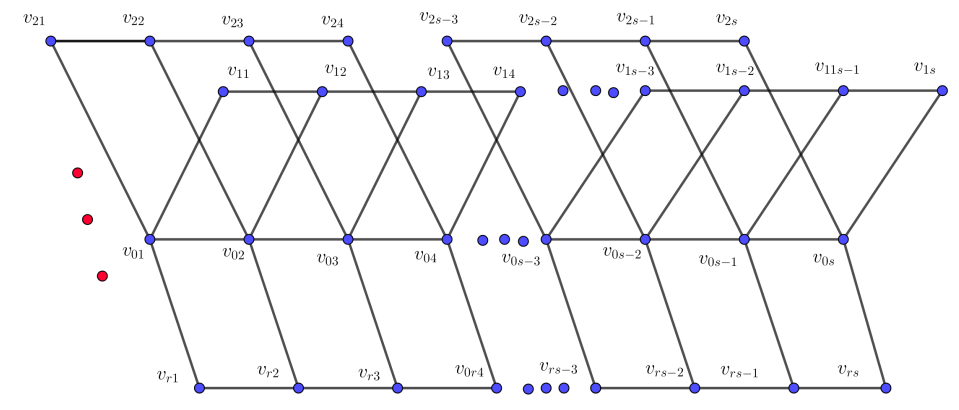

FIGURE 3. Stacked book graph

Let us partition the vertex set of the graph $\mathrm{G}$ to following subsets: $S_{1}=\left\{v_{i 1}, v_{i s} ; 1 \leq i \leq r\right\}, S_{2}=\left\{v_{0 j} ; 1 \leq\right.$ $j<s\}, S_{3}=\left\{v_{1 j} ; 2<j<s\right\}, S_{4}=\left\{v_{i 2} ; 1 \leq i \leq r\right\}, S_{5}=\left\{v_{i j} ; 2 \leq i \leq r\right.$, and $\left.2<j<s\right\}$ and $S_{6}=\left\{v_{0 s}\right\}$. Define a coloring function $f: V(G) \longrightarrow\{1,2, \ldots, k\}$ such that: 


$$
f\left(v_{i j}\right)= \begin{cases}1, & \text { if } v_{i j} \in S_{1} \\ j+1, & \text { if } v_{i j} \in S_{2} \\ s+j-2, & \text { if } v_{i j} \in S_{3} \\ f\left(v_{01}\right), & \text { if } v_{i j} \in S_{4} \text { or } v_{i j}=v_{0 s} \\ f\left(v_{1 j}\right), & \text { if } v_{i j} \in S_{5} .\end{cases}
$$

Clearly, $f$ is a monotone $(2 s-3)$ coloring function. So

$$
\chi_{m o}(G) \leq(2 s-3) .
$$

The set $S_{2} \cup S_{3} \cup\left\{v_{11}\right\}$ is monotone clique set in $G$ and need $(2 s-3)$ colors for monotone coloring. Therefore,

$$
\chi_{m o}(G) \geq(2 s-3) .
$$

Hence by inequalities 3.5 and 3.6, we get,

$$
\chi_{m o}(G)=(2 s-3) .
$$

Lemma 3.1. Let $G$ and $H$ be any non trivial connected graphs and $G \square H$ is the Cartesian product. Then any two vertices $(u, v)$ and $(x, y)$ are monotone adjacent in $G \square H$, if one of the following satisfy:

(1) $x=u$ in $G$ and $y$ is monotone adjacent with $v$ in $H$.

(2) $x$ and $u$ are monotone adjacent vertices in $G$ and $y=v$ in $H$.

(3) $x$ is monotone adjacent with $u$ in $G$ and $y$ is monotone adjacent with $v$ in $H$.

Theorem 3.4. For any two graphs $G$ and $H$,

$$
\chi_{m o}(G)+\chi_{m o}(H)-1 \leq \chi_{m o}(G \square H) \leq \chi_{m o}(G) \chi_{m o}(H) .
$$

Proof. Let $\chi_{m o}(G)=k_{1}$ and $\chi_{m o}(H)=k_{2}$. By Theorem 2.3, $\omega_{m o}(G)=k_{1}$ and $\omega_{m o}(H)=k_{2}$. Then there exist two monotone cliques: $C_{1}=\left\{u_{1}, u_{2}, \ldots, u_{k_{1}}\right\}$ and $C_{2}=\left\{v_{1}, v_{2}, \ldots, v_{k_{2}}\right\}$ in $G$ and $H$ respectively, with $k_{1}$ and $k_{2}$ vertices. Therefore, $\left\{\left(u_{1}, v_{1}\right),\left(u_{2}, v_{1}\right),\left(u_{3}, v_{1}\right), \ldots,\left(u_{k_{1}}, v_{1}\right),\left(u_{k_{1}}, v_{2}\right),\left(u_{k_{1}}, v_{3}\right), \ldots,\left(u_{k_{1}}, v_{k_{3}}\right)\right\}$ is monotone clique in $G \square H$ with $k_{1}+k_{2}-1$ vertices. Therefore,

$$
k_{1}+k_{2}-1 \leq \omega_{m o}(G \square H)=\chi_{m o}(G \square H) .
$$

Then,

$$
\omega_{m o}(G)+\omega_{m o}(H)-1 \leq \chi_{m o}(G \square H)
$$

Now, assume that $\chi_{m o}(G \square H)=m$, that means $\omega_{m o}(G \square H)=m$, then there exists at least one monotone clique set with $m$ vertices, say $C=\left\{w_{1}, w_{2}, \ldots, w_{m}\right\}$. By definition of Cartesian product any two adjacent 
vertices $w_{i}=\left(a_{i}, b_{i}\right), w_{i+1}=\left(a_{i+1}, b_{i+1}\right)$ either $a_{i} a_{i+1}$ is connected in $G$ with $b_{i}=b_{i+1}$ or $b_{i} b_{i+1}$ is connected in $H$ with $a_{i}=a_{i+1}$. Any sequence of adjacent vertices on $C$ which they are mutually different in first component will make correspondence monotone clique in $G$. Similarly, any sequence of adjacent vertices which they are mutually different in second component will make correspondence monotone clique in $H$. Let the maximum number of vertices for the correspondence monotone clique in $G$ which correspondence vertices in $G \square H$ is $s$ and similarly, the maximum number of vertices for the correspondence monotone clique in $H$ which correspondence vertices in $G \square H$ is $t$. Then the number of vertices on $C=\left\{w_{1}, w_{2}, \ldots, w_{m}\right\}$ will be at most $m=s t$, since $s \leq \omega_{m o}(G)$ and $t \leq \omega_{m o}(H)$. Then

$$
\omega_{m o}(G \square H) \leq \omega_{m o}(G) \omega_{m o}(H)
$$

Thus, by inequalities 3.7 and 3.8 ,

$$
\chi_{m o}(G)+\chi_{m o}(H)-1 \leq \chi_{m o}(G \square H) \leq \chi_{m o}(G) \chi_{m o}(H)
$$

Conflicts of Interest: The author(s) declare that there are no conflicts of interest regarding the publication of this paper.

\section{REFERENCES}

[1] D.M. Cardoso, J.O. Cerdeira, J.P. Cruz, C. Dominic, Injective Edge Chromatic Index of a Graph, ArXiv:1510.02626 [Math]. (2015).

[2] J.L. Fouquet, J.L. Jolivet, Strong edge-colorings of graphs and applications to multi k -gons, Ars Combin. 16A (1983), 141-150.

[3] F. Guthrie. Note on the colouring of maps. Proc. R. Soc. Edinburgh. 10 (1880), 727-728.

[4] I. Gutman, Kragujevac trees and their energy, Sci. Publ. State Univ. Novi Pazar Ser. A., Appl. Math. Inform. Mech. 6 (2) (2014), 71-79.

[5] G. Hahn, J. Kratochvíl, J. širán̆ and D. Sotteau, On the injective chromatic number of graphs. Discrete Math. 256 (2002), $179-192$.

[6] F. Harary, Graph theory, Addison-Wesley, Reading Mass. (1969).

[7] J.X. Li, J.M. Guo, W.C. Shiu, On the second largest Laplacian eigenvalues of graphs, Linear Algebra Appl. 438 (2013), $2438-2446$.

[8] N. Zufferey, P. Amstutz, P. Giaccari, Graph colouring approaches for a satellite range scheduling problem, J. Schedul. 11 (4) (2008), 263-277. 